\title{
Fotobiomodulação no reparo tecidual de lesão por pressão em região glútea: relato de experiência
}

\begin{abstract}
RESUMO | Objetivo: Avaliar a evolução clínica no reparo da lesão por pressão em região glútea à direita, por meio da fotobiomodulação e terapia fotodinâmica. Método: Trata-se de um estudo descritivo analítico experimental, através do relato de relato de experiência, desenvolvido no período de 26/01/2019 a 11/06/2019. Resultados: $O$ tratamento teve início no dia $26 / 01 / 2019$, lesão medindo $9,0 \mathrm{~cm}$ de comprimento, $5,0 \mathrm{~cm}$ de largura e 3,5 cm de profundidade, com a seguinte conduta nas primeiras 16 sessões: Terapia Fotodinâmica e cobertura primária de espuma impregnada com Polihexametileno de Biguanida, e secundária sempre que necessário. Após, foram realizadas sessões de laser pontual no leito da lesão e região perilesional, mantendo como cobertura primária gaze impregnada com Polihexametileno de Biguanida, e trocas realizadas sempre que necessário até o término do tratamento. Conclusão: Conclui-se que houve melhora no processo de cicatrização da lesão tratada com fotobiomodulação e terapia fotodinâmica, porém, é importante que o profissional esteja habilitado para atuar com essa prática.
\end{abstract}

Descritores: Laser de baixa potência; Terapia fotodinâmica; Lesão por pressão.

\begin{abstract}
Evaluate the clinical evolution in the repair of pressure injury in the right gluteal region, through photobiomodulation and photodynamic therapy. Method: This is an experimental analytical descriptive study, through the report of an experience report, developed from 01/26/2019 to 06/11/2019. Results: The treatment began on 01/26/2019, with a lesion measuring $9.0 \mathrm{~cm}$ in length, $5.0 \mathrm{~cm}$ in width and $3.5 \mathrm{~cm}$ in depth, with the following conduct in the first 16 sessions: Photodynamic Therapy and primary coverage of foam impregnated with Polyhexamethylene Biguanide and would second whenever necessary. Afterwards, punctual laser sessions were carried out in the wound bed and perilesional region, keeping gauze impregnated with Polyhexamethylene Biguanide as primary coverage, and exchanges were carried out whenever necessary until the end of the treatment. Conclusion: It is concluded that there was an improvement in the healing process of the lesion treated with photobiomodulation and photodynamic therapy, however, it is important that the professional is qualified to work with this practice.
\end{abstract}

Keywords: Breastfeeding; Nursing; Weaning; Maternal and Child Nursing;

RESUMEN | Objetivo: Evaluar la evolución clínica en la reparación de la lesión por presión en la región glútea derecha, mediante fotobiomodulación y terapia fotodinámica. Método: Se trata de un estudio descriptivo analítico experimental, a través del reporte de un relato de experiencia, desarrollado del 26/01/2019 al 06/11/2019. Resultados: El tratamiento se inició el 26/01/2019, con una lesión de $9,0 \mathrm{~cm}$ de largo, 5,0 cm de ancho y 3,5 cm de profundidad, con la siguiente conducta en las primeras 16 sesiones: Terapia Fotodinámica y cobertura primaria de espuma impregnada con Polihexametilen biguanida y segundo cuando sea necesario. Posteriormente se realizaron sesiones puntuales de láser en el lecho de la herida y región perilesional, manteniendo una gasa impregnada con Polihexametilen Biguanida como cobertura primaria, y se realizaron intercambios cuando fue necesario hasta el final del tratamiento. Conclusión: Se concluye que hubo una mejora en el proceso de curación de la lesión tratada con fotobiomodulación y terapia fotodinámica, sin embargo, es importante que el profesional esté calificado para trabajar con esta práctica.

Palabras claves: : Láser de baja potencia; Terapia fotodinámica; Lesión por presión.

\section{Keli Cristina Ferreira}

Enfermeira, Mestranda em Gestão do Cuidado em Saúde ,Pós graduanda em Estomaterapia pelo Centro Universitário São Camilo, Professora de Pós Graduação pela Revolution-Instituicao de Ensino. ORCID:/0000-0003-2049-9585

\section{João Júnior Gomes}

Graduado em Enfermagem, Mestre em Promoção da Saúde, Doutor em Ciências da Saúde, Pós graduado em Estomaterapia. ORCID: 0000-0003-4171-2752

Anelvira de Oliveira Florentino
Doutoranda em Enfermagem, Mestre em
Pesquisa Clínica pela Unesp/Botucatu, Pro-
fessora de Graduação na UNIFSP e FAESB
,Enfermeira especialista em Gestão em
Enfermagem, Pós Graduanda em Estoma-
terapia.
ORCID: 0000-0001-8628-0565

ORCID: 0000-0001-8628-0565

Recebido em: 17/06/2021

Aprovado em: 23/07/2021
INTRODUÇÃO

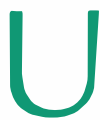
ma forma de tratamento não invasivo para auxiliar no fechamento de feridas em Lesão por Pressão (LPP), que vem aumentando interesse na área médica e afins, é a Terapia com Laser em Baixa Intensidade (LBI), Fotobiomodulação (FBM) ou simplesmente, Laserterapia. Esta nova característica terapêutica vem sendo empregada atualmente, além da sua eficiente ação anti-inflamatória, analgésica. ${ }^{1}$ 
O uso do laser deu início na Europa entre as décadas de 60 e 70, onde foram analisados resultados significativos durante o processo de cicatrização da lesão. Ainda que, alguns estudos comprovem a eficácia do uso do laser em baixa intensidade em lesões, é importante ressaltar, uma instabilidade nos estudos referentes à falta de protocolos para o procedimento. $^{2}$

O LBI apresenta um comprimento de onda específico, correspondente ao pico de absorção do fotossensibilizante (FS). Sua capacidade de emissão de luz monocromática de alta fluência associada à precisão do foco permite tratar pequenas lesões com mínimo dano ao tecido ao redor, em um curto intervalo de tempo. Gerando um aumento na propagação das células reparativas, além da renovação do colágeno e ajudando no processo de reparação tecidual. Mas, para que essa reparação tecidual ocorra, é importante que a infecção na lesão esteja controlada. ${ }^{3}$

Pode-se destacar a Terapia Fotodinâmica (PDT) como uma terapia essencial aos procedimentos antimicrobianos. A PDT é uma reação química ativada por luz usada para destruição seletiva de um tecido e requer um agente FS no tecido-alvo, uma fonte de luz e oxigênio. ${ }^{4,5}$

O Azul de Metileno (AM) é um FS que proporciona um ótimo resultado para a aplicação clínica da PDT devido ao seu comprimento de absorção máximo ser em torno de $660 \mathrm{~nm}$, possuindo uma boa penetração na pele, ação contra bactérias, flexibilidade e baixa toxicidade.(6)

Existem atualmente no mercado muitos produtos para tratar feridas, o que torna a escolha do tratamento correto uma tarefa difícil e desafiadora. Nesse sentido, alguns preceitos devem ser considerados como fatores relacionados com a ferida e a pele adjacente: etiologia, tamanho, profundidade, localização anatômica, volume de exsudato, risco ou presença de infecção, condições da pele adjacente; condições nutricionais, doenças de base entre outros. ${ }^{7}$

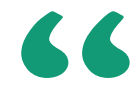

\section{A PDT é uma reação química ativada por luz usada para destruição seletiva de um tecido e requer um agente FS no tecido-alvo, uma fonte de luz e oxigênio.}

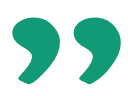

Esse avanço do conhecimento no tratamento de feridas também contribuiu para que profissionais de saúde envolvidos neste cuidado pudessem revisar conceitos e práticas, e reconhecer que a lesão é apenas mais um aspecto dentro de um todo, que é o ser humano. ${ }^{8}$

Os tratamentos convencionais, muitas vezes, acabam prolongando o tempo de cicatrização destes tipos de lesões, o que ocasionam altos custos para os tratamentos, além disso, a qualidade de vida dos pacientes acometidos por lesões é extremamente comprometida pela dor, dificuldade de locomoção, processo infeccioso crônico, uso de medicamentos prolongados e a condição psicológica.(9)

Enquanto que a FBM é uma técnica indolor que consiste na doação de energia luminosa para o tecido lesionado. Esta terapia tem sido utilizada nas diversas áreas da saúde e tem como ação acelerar a cicatrização tecidual, modular a inflamação, promover analgesia e as complicações recorrentes ao longo do processo de reparação tecidual. ${ }^{8,9}$

Devido a estas ações específicas, a FBM tem se caracterizado como um poderoso anti-inflamatório, cujas vantagens sobre os medicamentos convencionais são inúmeras, principalmente pela ausência de efeitos colaterais, ação local, baixo custo e grande aceitabilidade pelos pacientes. A FBM pode ser associada a qualquer outro método de tratamento. $\mathrm{O}$ número de sessões varia de acordo com o tipo e tamanho da lesão. ${ }^{8}$

Já a terapia fotodinâmica possui alguns benefícios em lesão por pressão, podendo favorecer o prognóstico, reduzindo a carga microbiana, consequentemente, produzindo uma melhoria e acelerando o reparo tecidual, propiciando ao paciente uma melhor da qualidade de vida. ${ }^{10}$

A cicatrização de feridas consiste em uma perfeita e coordenada cascata de eventos celulares e moleculares que interagem para que ocorra a reconstrução do tecido. ${ }^{11}$ Após um ferimento, inicia-se uma sequência desses eventos biológicos visando promover o reparo da lesão, processo caracterizado como cicatrização. ${ }^{12}$

A cicatrização é formada por três fases: fase inflamatória, fase proliferativa e fase de remodelação. Tal evento é um processo dinâmico que envolve fenômenos bioquímicos e fisiológicos que se comportam de forma harmoniosa a fim de garantir a restauração tecidual. ${ }^{9}$

As ações da PDT e da FBM, como terapias coadjuvantes ao protocolo de acelerar a cicatrização tecidual, são muito importantes, pois evitam o agravamento da lesão, colonização e infecção por bactérias. Este relato baseou-se na seguinte questão norteadora: "Qual a evolução clínica no reparo da lesão por pressão em região glútea, por meio da PDT e FBM?". 
Diante ao exposto, o presente estudo tem por objetivo avaliar a evolução clínica no reparo da lesão por pressão em região glútea, por meio da PDT e FBM, através de um relato de experiência.

\section{MÉTODO}

Trata-se de um estudo descritivo analítico experimental, do tipo relato de experiência, desenvolvido no período de 26/01/2019 a 11/06/2019 que relata o tratamento da LPP de um paciente que esteve hospitalizado por duas vezes.

Sua primeira internação se deu em 05/12/2018, ficando vinte e nove dias hospitalizado, sendo treze dias na Unidade de Terapia Intensiva (UTI), e sua segunda internação ficou apenas quatro dias. Somente após alta hospitalar, os familiares do paciente fizeram contato, procurando o serviço especializado para atendimento Home Care.

Como trata-se de um relato de experiência, dispensa-se o envio do projeto de pesquisa a um Comitê de Ética em Pesquisa por se tratar do relato de uma experiência, uma assistência realizada. A fim de respeitar as normas éticas em pesquisa e publicação científica, o responsável pelo paciente assinou o Termo de Consentimento Livre e Esclarecido autorizando a divulgação do relato no meio acadêmico através de publicações e apresentações em eventos científicos, assim como o uso das imagens da lesão e processo de cicatrização. Enfatiza-se o respeito ao paciente e sua família, assim como o sigilo de quaisquer informações que vão contra o anonimato.

Foi realizada a coleta do histórico do paciente e exame físico para mensuração da lesão em centímetros e registro fotográfico, em seguida foram realizadas as intervenções necessárias.

C.S.S. Jr. sexo masculino, 61 anos, apresentando LPP em região glútea à direita, ex-tabagista, portador de doença renal crônica, não dialítico, hipertenso, hipocorado ++/4+, emagrecido $46 \mathrm{~kg}$, lesão com sinais de infecção, apresentando

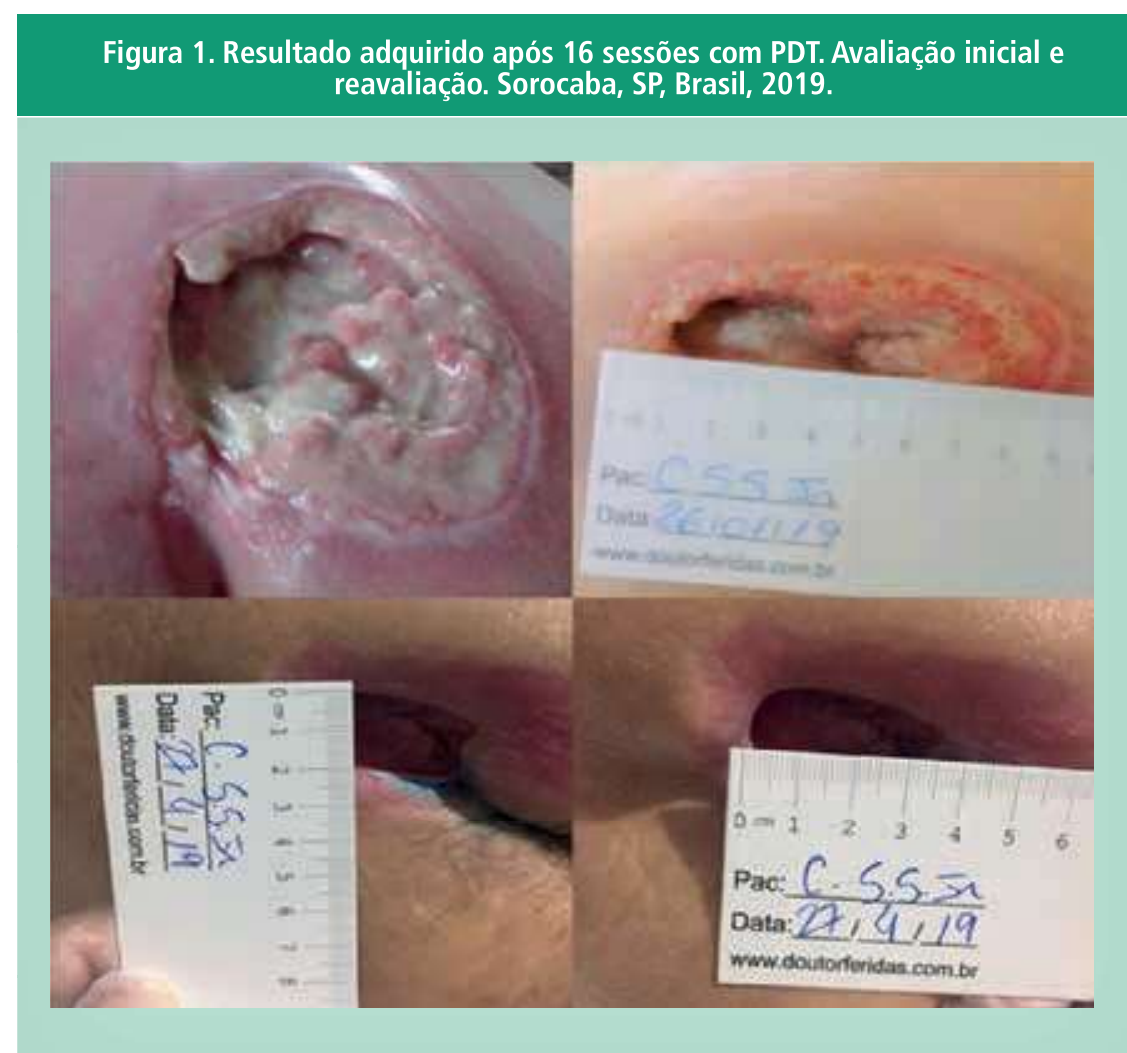

Fonte: Acervo pessoal, 2019.

Figura 2. Resultado adquirido no final do tratamento. Sorocaba, SP, Brasil, 2019

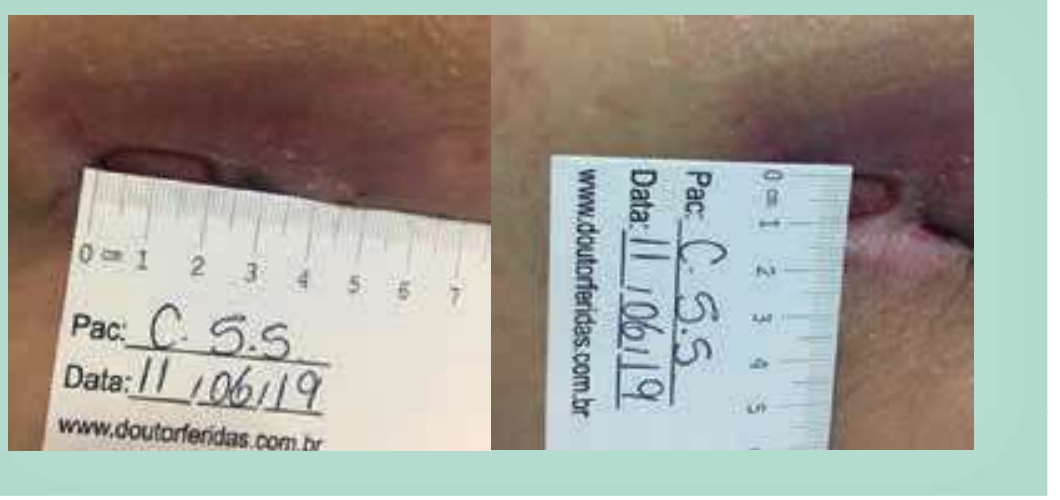

Fonte: Acervo pessoal, 2019

esfacelo aderido e exsudação moderada. Lesão medindo $9,0 \mathrm{~cm}$ de comprimento $X$ 5,0 cm de largura $X 3,5 \mathrm{~cm}$ de profundidade. A mensuração foi realizada com o auxílio de uma fita métrica milimetrada, medindo-a em seu maior comprimento e largura. Para mensurar a profundidade, foi utilizado uma sonda uretral de calibre no 8 com o auxílio de uma fita métrica milimetrada. As medidas foram anotadas, fotografadas, e posteriormente analisadas com a da última sessão. Paciente foi hospitalizado pela primeira vez entre os dias 05/12/2018 a 04/01/2019, sendo que entre os dias 6/12/2018 a 19/12/2018 necessitou ser intubado; e, pela segunda vez, foi internado entre os dias 18/01/2019 a 22/01/2019. Recebeu 
alta hospitalar com LPP em região glútea à direita.

O equipamento utilizado foi o apareIho de Laser DMC Therapy XT de baixa potência $100 \mathrm{~mW}-660 \mathrm{~nm}$.

Após avaliação, iniciou-se o tratamento com PDT duas vezes por semana, ininterruptas totalizando dezesseis intervenções. Em seguida, manteve o tratamento uma vez na semana com fotobiomodulação e coberturas de alta tecnologia até o término.

\section{Relato da experiência}

O tratamento proposto foi a Fotobiomodulação e cobertura de alta tecnologia.

Nas primeiras dezesseis sessões, os procedimentos foram realizados duas vezes na semana, com as seguintes condutas: Lavagem da lesão com solução de Polihexametileno de Biguanida (PHMB) deixando agir por 15 minutos. Posteriormente, lavagem com solução fisiológica (SF) à $0,9 \%$, retirando todo resíduo do $\mathrm{PHMB}$ e secagem com gazes estéril. Para realizar a PDT, utilizamos como fotossensibilizante a solução comercial de AM a $0,01 \%$ quantidade suficiente para cobrir a extensão da lesão. Para proteger a região perilesional optou-se pelo creme de barreira, como cobertura primária espuma impregnada com PHMB, e como cobertura secundária gaze estéril, sendo trocadas sempre que necessário pela esposa do paciente, que foi orientada e treinada pela enfermeira.

As quatro primeiras sessões de PDT foram realizadas com densidade de energia por ponto à $9 \mathrm{~J}$ aules $(\mathrm{J}) / \mathrm{cm}^{2}$. E as sete sessões posteriores com densidade de energia por ponto à $6 \mathrm{~J} / \mathrm{cm}^{2}$, como verifica-se na Figura 1.

Após as primeiras dezesseis sessões, as intervenções passaram a acontecer uma vez na semana, mantendo a mesma conduta anterior, porém a FBM foi realizada com densidade de energia por ponto à $2 \mathrm{~J} / \mathrm{cm}^{2}$ no leito da lesão e na região perilesional $4 \mathrm{~J} / \mathrm{cm}^{2}$ infravermelho, como verifica-se na Figura 2

É visível que esses tratamentos coadjuvantes (PDT/FBM) ajudam no processo de cicatrização da lesão crônica, constatando pela diminuição das dimensões da lesão, redução da infecção e dor, reepitelização, diminuição do custo e tempo de tratamento. Porém, é de grande importância que a enfermagem seja capacitada para executar essa prática.(13)

\section{DISCUSSÃO}

Analisando a eficácia da aplicação do LBI na cicatrização de lesão por pressão, além de várias patologias que podem ser estudadas com essa terapia, é necessário que a enfermagem seja habilitada e aprimore os conhecimentos em FBM. ${ }^{13}$

O Conselho Federal de Enfermagem (Cofen) destaca o importante papel da enfermagem no tratamento das feridas, e é fundamental o aperfeiçoamento científico nesta área para desenvolver o empoderamento nessa tecnologia. ${ }^{14}$

O Parecer Cofen n. ${ }^{\circ} 13 / 2018(14)$ ressalta que a Câmara Técnica não encontrou óbices na utilização da laserterapia com autonomia pelo Enfermeiro, após estar devidamente capacitado para realizar a prática.

É sabido que a LPP prolonga a hospitalização e aumenta o risco para o desenvolvimento de outras complicações como infecções, prejudicando a boa evolução do indivíduo e aumentando os custos financeiros. ${ }^{15}$

A FBM é uma técnica capaz de acelerar o processo de reparação de tecidos biológicos traumatizados. Os mecanismos que envolvem o processo de bioestimulação ocorrem em nível molecular. A luz laser penetra no interior do tecido onde é absorvido por determinados cromóforos, resultando no aumento do metabolismo celular, elevando a motilidade de células epiteliais, a quantidade de tecido de granulação e, podem diminuir a síntese de mediadores inflamatórios. 5,16

O paciente do presente estudo foi submetido a intervenções com Lasertera- pia e obteve redução na área total da ferida, resultado semelhante a estudo, onde uma das lesões submetidas ao tratamento, obteve $50 \%$ de cicatrização após dez aplicações.?

Em um outro estudo de caso, a LPP em região lombo sacral apresentou uma cicatrização total, após a trigésima intervenção, é possível observar a presença de extensa área coberta por tecido cicatricial, entretanto esta havia sido reparada totalmente na vigésima segunda intervenção, sendo a área necrosada eliminada do local na décima intervenção. ${ }^{17}$

É possível encontrar em alguns estudos a efetividade do uso do LBI na cicatrização de LPP. A técnica da dosimetria varia conforme as características das feridas, levando-se em conta o aspecto do leito e, principalmente, sua dimensão.

O profissional da área da saúde deve conhecer bem a fundamentação teórica da laserterapia, além das funcionalidades do laser, tais como: modos de emissão (contínuo ou pulsado), duração do pulso, comprimento de onda e densidade de energia. Utilizando os parâmetros corretos, é possível realizar um tratamento eficaz e obter o máximo dos benefícios terapêuticos ${ }^{18}$.

Neste estudo, o paciente alcançou resultados rápidos de cicatrização após longo período de instalação da LPP, a qual the ocasionava muitos incômodos e restringia suas atividades de vida diária. Recomendamos que outros estudos continuem sendo analisados, com maior número de pessoas e com outros métodos, para que o assunto laserterapia e LPP consigam ser melhor entendidas e determinem os benefícios desta terapia quanto à cicatrização de lesão por pressão.

\section{CONCLUSÃO}

Diante da melhora no processo de cicatrização da lesão, a terapia com LBI vem demonstrando muitos benefícios. Assim, a partir deste relato de experiência, constatou-se que essa técnica proporciona efeito cicatrizante, favorecendo 
a restauração dos tecidos, é um agente anti-inflamatório, reduzindo a dor e o inchaço característico da lesão de pele.

No que se refere à atuação da enfermagem, a mesma está apta a realizar tratamento e diagnóstico das lesões de pele/feridas. No entanto, para aplicação do $L B \mid$ o profissional precisa manter-se em qualificação contínua, para adquirir habilidades e competências para uso do recurso terapêutico.

Enfatiza-se o amplo leque de espe- cialidades disponíveis para atuação do enfermeiro, tendo, por sua vez, cada vez maior autonomia a partir de especializações e atualizações. A Ciência move a Saúde, e a capacitação move o enfermeiro a alcançar o lugar que lhe é de direito.

\section{Referências}

1. Moura JPG, Brandão LB, Barcessat ARP. Estudo da Terapia Fotodinâmica (PDT) no reparo de lesões teciduais: estudo de casos clínicos. Estação Científica (UNIFAP). 2018;8(1):103-110.

2. Salomé GM, Ferreira LM. Low intensity laser and microcurrent in scratching of skin wounds in rats. Journal of Nursing UFPE on line. 2017;11(9):3567-3573.

3. Haley D, Pratt O. Basic Principles of Lasers. Elsevier; 2017.

4. Soukos N S, et al. Photodynamic therapy for endodontic disinfection. J Endod. 2006;32(10):979-984

5. Peng Q, Juzeniene A, Chen J, Svaasand LO, Warloe T, Giercksky KE, Moan J. Lasers in Medicine. UK: IOP Publishing; 2008.

6. Longo JPF, Azevedo RB. Efeito da terapia fotodinâmica mediada pelo azul de metileno sobre bactérias cariogênicas. Rev. Clín. Pesq. Odontol. 2010;3:249257.

7. Lopes LDF. Utilização do laser de $660 \mathrm{~nm}, 17 \mathrm{~J} / \mathrm{cm} 2$ em úlceras por pressão - um relato de caso. Rev. Neurocienc. 2011;19(4):668-674.

8. Petz FC. Terapia a laser na cicatrização da úlcera por pressão em adultos e idosos: revisão sistemática. Curitiba, 2015. 140 f. Pós-Graduação em Enfermagem, Setor de Ciências da Saúde, Universidade Federal do Paraná, (Dissertação) Mestre. Área de concentração: Prática Profissional de Enfermagem. 2015.

9. Bourguignon Filho AM, Feitosa ACF, Beltrão GC, Pagnoncelli GC. Utilização do laser de baixa intensidade no processo de cicatrização tecidual. Revisão de literatura. Rev. Port. Estomatol Cir. Maxilofac. 2005;46(1):37-43.

10. Araújo AR, Chaves MEA, Tizon AFF, Silva DB, Piantino LL, Azevedo VS. Tratamento de úlceras de decúbito com laser de baixa intensidade. Fisioterapia Brasil. 2009;10(2):135-143.

11. Mandelbaum SH, Di Santis EP. Cicatrização: conceitos atuais e recursos auxiliares - Parte I* An. Bras. Dermatol. 2003;78(4):393-410.

12. Dealey C. Cuidando de feridas: um guia para as enfermeiras. Tradução: Rúbia Aparecida Lacerda, Vera Lucia Conceição Gouveia Santos. 3.ed. São Paulo: Atheneu; 2008

13. Armelin MVAL, Saraiva KVO, Corazza AV, Silva GD, Jurado SR, Sanchez A. 0 uso do laser de baixa potência por enfermeiro no tratamento de lesões cutâneas e orais. Rev. Nursing (Säo Paulo). 2019;22(253):3006-3010.

14. Conselho Federal de Enfermagem (Cofen). Parecer n. ${ }^{\circ}$ 13/2018 Cofen CTLN. Legislação Profissional: questionamento de profissional acerca do uso de laserterapia de baixa intensidade em lesões mamilares. Brasília (DF): Cofen; 2018.

15. Sousa RG, Oliveira TL, Lima LR, Stival MM. Fatores associados à úlcera por pressão (UPP) em pacientes críticos: revisão integrativa da literatura. Univ Ci Saúde. 2016; 14(1):77-84.

16. Weber MH. The Intravenous Laser Blood Irradiation - Introduction of a New Therapy. Medical Center, Lauenförde, Germany; 2009.

17. Ramos LAV, Brito MM, Queiroz WWM, Santos D, Dias CAGM, Oliveira JCS, et al. A eficácia do laser de baixa potência na cicatrização de úlcera de decúbito em paciente diabético: estudo de caso. Biota Amazônica. 2014;4(2):74-76.

18. Busnardo VL, Simões MLPSB. Os efeitos do laser hélio-neônio de baixa intensidade na cicatrização de lesões induzidas em ratos. Rev Bras Fisioter. 2010:14(1):45-51. 